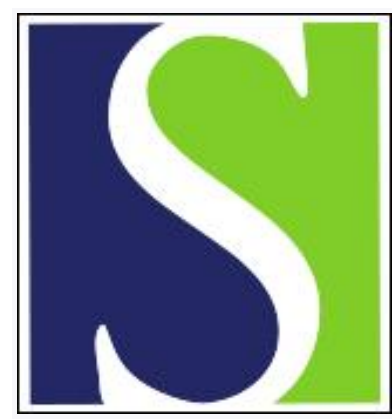

Scand J Work Environ Health 2018;44(1):1-2

https://doi.org/10.5271/sjweh.3699

Published online: 11 Dec 2017, Issue date: 01 Jan 2018

Water, rest, shade: Can simple actions stop an epidemic of chronic kidney disease of unknown etiology among farm workers in Central America?

by Broberg $\mathrm{K}$

Affiliation: Institute of Environmental Medicine, Karolinska Institutet, Stockholm, Sweden. karin.broberg@ki.se

Refers to the following text of the Journal: 2018;44(1):16-24

Key terms: Central America; chronic kidney disease; CKD; dehydration; editorial; El Salvador; farm worker; farmer; kidney damage; kidney disease; Mesoamerican nephropathy; rest; shade; sugarcane worker; water

This article in PubMed: www.ncbi.nlm.nih.gov/pubmed/29226298 


\section{Water, rest, shade: Can simple actions stop an epidemic of chronic kidney disease of unknown etiology among farm workers in Central America?}

Chronic kidney disease (CKD) of unknown etiology (CKDu) was first reported in El Salvador and Nicaragua in Central America, where it has very high prevalence and mortality. However, CKDu is now an epidemic in countries around the equator: Central America and Mexico, India and Sri Lanka, and Egypt. Unlike the more common kind of CKD, seen mostly among the elderly in urban areas, CKDu appears to be a rural disease, affecting farm workers, mostly men aged 30-50 years. This disease has substantial mortality, and as it prevents sick men and women from participating in the workforce and limits their role in home life, CKDu also severely affects society.

CKDu is so fatal in part because it is hard to detect. In the early stages of the disease, the patients show no symptoms; by the time a diagnosis is made, the damage to the kidneys is irreversible, leading to high blood pressure, weakness, sleep problems, nausea and vomiting, and weight loss. Access to dialysis or kidney transplant is often very limited and costly in the areas where the disease is prevalent. Many patients do not survive a long time between diagnosis and death.

In Central America, which has been hit the hardest, the leading hypothesis is that CKDu is an occupational disease, caused by long hours of work in the heat with too little drinking water, leading to chronic dehydration, such as may occur during work in the cane fields. Sugarcane cutting is a repetitive highintensity work, and workers spend the majority of their work day above $50 \%$ of their maximal heart rate (1). An animal study showed that recurrent dehydration can induce renal injury (2), and a human pilot study indicated a possible mechanism behind chronic dehydration and kidney damage: high levels of uric acid crystals were found in cane cutters' urine, especially at the ends of their shifts (3). Those crystals may injure the kidneys. However, other causal factors have been debated, such as metals and silica in the drinking water or pesticides used in farming $(4,5)$. It is possible that the disease is multifactorial, depending on the region. However, a recent review of CDKu and use of pesticides did not find strong evidence for an association (6).

In this issue of the Scandinavian Journal of Work, Environment \& Health, Wegman et al (7) present the results of an intervention study where they assessed the potential to reduce kidney damage, as measured by biomarkers of dehydration, kidney function, and muscle-tissue breakdown in serum and urine. They studied the implementation of a water, rest, shade, and efficiency intervention program among sugarcane workers in El Salvador. The program began two months into the 5-month harvest: one of two groups of sugarcane workers was provided with portable water reservoirs, mobile shaded tents, and scheduled rest periods (10-15 minutes of rest every $1-1.5$ hours). Health data, blood, and urine samples were collected at baseline and at three subsequent times over the course of the harvest.

The intervention study found that over the course of the working day, urine osmolality increased, indicating dehydration, and the estimated glomerular filtration rate (eGFR), reflecting kidney function, decreased. The decrease in eGFR across the day was present in both groups, but smaller for the intervention group. Decreased eGFR over the 5-month harvest was also seen in both groups, but was $56 \%$ higher in the group not receiving the intervention $\left(-5.3\right.$ versus $\left.-3.4 \mathrm{~mL} / \mathrm{min} / 1.73 \mathrm{~m}^{2}\right)$. Moreover, the decrease in eGFR seemed to cease after the start of the intervention in the group receiving the program. No long-term effects on the other biomarkers were found. 
Theo Bodin, the senior author of the study (7) explains the study's biggest challenge was to recruit the cane workers and to plan and coordinate the study with the employers and the workers. Security was also a major issue in performing such a study as El Salvador is considered one of the 20 most dangerous countries in the world. The authors should be applauded for collecting and handling biological samples under these conditions, as the measurement of biomarkers in serum and urine provides solid data on positive effects of the intervention. Furthermore, the study gives additional support to the notion that heat stress and/or repeated dehydration causes the disease in this region.

The study has limitations that reflect the difficulties in performing such a study in this region. A limited numbers of individuals participated, there were dropouts during the study, and there are possible confounders and unmeasured risk factors that need to be considered. Still, the effects of the intervention on kidney function and damage were evident, suggesting a clear effect of the apparently simple actions of providing shade, rest, and access to water. Whether these actions can prevent CKDu in El Salvador, or elsewhere, is not yet clear and needs further research.

This intervention study has received a lot of attention in El Salvador, and as a result, the sugarcane industry has started to improve the work environment for cane workers in different countries. The intervention program is continuing in El Salvador and intervention studies have started in other Central American countries, such as Nicaragua, says Theo Bodin. Obviously, there is a desperate need to identify the causes for this fatal occupational disease and find ways to prevent it. This study by Wegman and co-authors provides evidence for how further actions should be taken.

\title{
References
}

1. Lucas RA, Bodin T, García-Trabanino R, Wesseling C, Glaser J, Weiss I, et al. Heat stress and workload associated with sugarcane cutting-an excessively strenuous occupation! Extrem Physiol Med 2015;4(1):1. https://doi.org/10.1186/20467648-4-S1-A23

2. Roncal Jimenez CA, Ishimoto T, Lanaspa MA, Rivard CJ, Nakagawa T, Ejaz AA, et al. Fructokinase activity mediates dehydration-induced renal injury. Kidney Int. 2014;86(2):294-302. https://doi.org/10.1038/ki.2013.492

3. Roncal-Jimenez C, García-Trabanino R, Barregard L, Lanaspa MA, Wesseling C, Harra T, et al. Heat Stress Nephropathy From Exercise-Induced Uric Acid Crystalluria: A Perspective on Mesoamerican Nephropathy. Am J Kidney Dis 2016;67(1):20-30. https://doi.org/10.1053/j.ajkd.2015.08.021

4. Mascarenhas S, Mutnuri S, Ganguly A. Deleterious role of trace elements - Silica and lead in the development of chronic kidney disease. Chemosphere 2017;177:239-249. https://doi.org/10.1016/j.chemosphere.2017.02.155

5. Jayasumana C, Paranagama P, Agampodi S, Wijewardane C, Gunatilake S, Siribaddana S Drinking well water and occupational exposure to Herbicides is associated with chronic kidney disease, in Padavi-Sripura, Sri Lanka. Environ Health 2015;18;14:6. https://doi.org/10.1186/1476-069X-14-6

6. Valcke M, Levasseur ME, Soares da Silva A, Wesseling C. Pesticide exposures and chronic kidney disease of unknown etiology: an epidemiologic review. Environ Health 2017;16(1):49. https://doi.org/10.1186/s12940-017-0254-0

7. Wegman DH, Apelqvist J, Bottai M, Ekström U, García-Trabanino R, Glaser J, et al. The Work Health and Efficiency (WE) Program Working Group. Intervention to diminish dehydration and kidney damage among sugarcane workers. Scand J Work Environ Health. 2018;44(1):16-24. https://doi.org/10.5271/sjweh.3659.

\author{
Karin Broberg, Professor, PhD \\ Institute of Environmental Medicine \\ Karolinska Institutet \\ Stockholm, Sweden \\ [email: karin.broberg@ki.se]
}

